\title{
Edge-Ramsey Theory
}

\author{
K. Cantwell \\ 16601 Garfield Lot \#101, Paramount, \\ CA 90723 , USA
}

\begin{abstract}
Let $K$ be a configuration, a set of points in some finite-dimensional Euclidean space. Let $n$ and $k$ be positive integers. The notation $R(K, n, r)$ is an abbreviation for the following statement: For every $r$-coloring of the points of the $n$-dimensional Euclidean space, $R^{n}$, a monochromatic configuration $L$ which is congruent to $K$ exists.

A configuration $K$ is Ramsey if the following holds: For every positive integer $r$, a positive integer $n=n(K, r)$ exists such that, for all $m \geq n, R(K, m, r)$ holds.

A configuration is spherical if it can be embedded in the surface of a sphere in $n$-space, provided $n$ is sufficiently large. It is relatively easy to show that if a configuration is Ramsey, it must be spherical. Accordingly, a good fraction of the research efforts in Euclidean Ramsey theory is devoted to determining which spherical configurations are Ramsey. It is known that the $n$-dimensional measure polytopes (the higher-dimensional analogs of a cube), the $n$-dimensional simplex, and the regular polyhedra in $R^{2}$ and $R^{3}$ are Ramsey.

Now let $E$ denote a set of edges in a configuration $K$. The pair $(K, E)$ is called an edgeconfiguration, and $R_{e}(K, E, n, r)$ is used as an abbreviation for the following statement: For any $r$-coloring of the edges of $R^{n}$, there is an edge configuration $(L, F)$ congruent to $(K, E)$ so that all edges in $F$ are assigned the same color.

An edge-configuration is edge-Ramsey if, for all $r \geq 1$, a positive integer $n=n(K, E, r)$ exists so that if $m \geq n$, the statement $R_{e}(K, E, m, r)$ holds. If $K$ is a regular polytope, it is said $K$ is edge-Ramsey when the configuration determined by the set of edges of minimum length is edge-Ramsey.

It is known that the $n$-dimensional simplex is edge-Ramsey and that the nodes of any edge-Ramsey configuration can be partitioned into two spherical sets. Furthermore, the edges of any edge-Ramsey configuration must all have the same length. It is conjectured that the unit square is edge-Ramsey, and it is natural to ask the more general question: Which regular polytopes are edge-Ramsey?

In this article it is shown that the $\boldsymbol{n}$-dimensional measure polytope and the $\boldsymbol{n}$-dimensional cross polytope are edge-Ramsey. It is also shown that these two infinite families and the $n$-dimensional simplexes are the only regular edge-Ramsey polytopes, with the possible exceptions of the hexagon and the 24-cell.
\end{abstract}




\section{Introduction}

In [6], Erdös et al, noted the following facts:

Theorem 1.1 [6]. The k-dimensional simplex is edge-Ramsey.

Theorem 1.2 [6]. If an edge configuration $(K, E)$ is edge-Ramsey, each of the edges in $E$ must have the same length.

In [15] Graham proves the following results:

Theorem 1.3 [15]. If an edge configuration $(K, E)$ is edge-Ramsey, the endpoints of the edges in $E$ can be covered by the surfaces of two spheres.

Let $(K, E)$ be a configuration. Then let $G(K, E)$ denote the graph whose vertex set is $K$ and whose edge set is $E$.

Theorem 1.4 [15]. If $(K, E)$ is a configuration of line segments such that $K$ is not spherical and $G(K, E)$ is not bipartite, then $(K, E)$ is not edge-Ramsey.

In this dissertation when we say that a polytope is edge-Ramsey, we mean that the edge configuration determined by its edges of minimum length is edge-Ramsey. When we say a configuration is bispherical we mean that its vertices lie on two spheres, but no single sphere contains all its vertices. We prove the following:

1. The measure polytope, the $n$-dimensional analog of the cube, is edge-Ramsey (Theorem 2.1).

2. The cross polytope, the $n$-dimensional analog of the octahedron, is edge-Ramsey (Theorem 3.3).

3. If a configuration is edge-Ramsey when we allow similarity as well as congruence transformations, it is edge-Ramsey under congruence transformations alone (Theorem 5.4).

4. There is an edge-configuration $(K, E)$ with $K$ bispherical and $G(K, E)$ bipartite such that $(K, E)$ is not edge-Ramsey (Theorem 5.1).

5. The only regular polygons that can be edge-Ramsey are the triangle, the square, and the hexagon (Theorem 4.3).

6. We can obtain a complete classification of those regular polytopes which are edgeRamsey, except for the hexagon and the 24-cell (Section 4). These last two cases remain unsolved.

\section{All $n$-Dimensional Measure Polytopes are Edge-Ramsey}

Let $(K, E)$ be an edge-configuration. Recall that we use $R_{e}(K, E, n, r)$ as an abbreviation for the statement that, for any $r$-coloring of the edges of $R^{n}$, there is an edge configuration 
$(L, F)$ congruent to $(K, E)$ with all edges of $F$ assigned the same color. An edgeconfiguration $(K, E)$ is edge-Ramsey if, for all $r$, there is an $n$ such that statement $R_{e}(K, E, n, r)$ is true. Erdös et al. have asked if the square is edge-Ramsey [6]. The answer is yes. In this section we prove a generalization (Theorem 2.1) of this result.

To state the generalization we need two definitions. An $n$-dimensional measure polytope is the $n$-dimensional analog of the cube. We use the term $n$-dimensional measure polytope here to conform to the system of names of regular polytopes used by Coxeter in [4]. It is the convex hull of all points in $R^{n}$ with all coordinates equal to 0 or 1.

A face polytope of a convex polytope, $K$, is the intersection of $K$ and a nonempty set of the boundary hyperplanes of $K$. Of course, a face polytope of $K$ has smaller dimension than $K$. The face polytopes of an $n$-dimensional measure polytope with side $a$ are measure polytopes with side $a$ of dimension $n-1$ or less.

For example, a cube is a three-dimensional measure polytope. It has six face polytopes of dimension two and twelve face polytopes of dimension one.

Theorem 2.1. For all $r, n, m$, and $a$, there is a $p$ such that if the $m$-dimensional measure polytopes of side a whose vertices have coordinates equal to 0 and $a / 2^{1 / 2}$ are $r$-colored in $R^{p}$, there is an $n$-dimensional measure polytope of side a such that all its $m$-dimensional face polytopes are the same color.

Before giving the proof, we must state some facts about coordinate representations and prove a lemma. One way of representing the $2^{n}$ points of an $n$-dimensional measure polytope of side $a$ in any $s$-dimensional space where $s \geq 2 n$ is as follows. First pick out $2 n$ coordinates and arbitrarily fix the remaining $s-2 n$ coordinates. Then form $n$ pairs of consecutive coordinates from the $2 n$ coordinates. In each pair let one coordinate equal $a / 2^{1 / 2}$; let the other equal 0 .

Since there are two ways of assigning coordinates to each consecutive pair, there are $2^{n}$ such points. All $m$-dimensional face polytopes are sets of $2^{m}$ points whose coordinates are fixed in $n-m$ of the pairs and allowed to vary in the remaining $m$ coordinate pairs. Note that there is only one such polytope in $2 n$-dimensional space since there are no dimensions whose coordinates may be arbitrarily fixed.

Call the pairs of coordinates allowed to vary moving coordinate pairs. Call a measure polytope with this coordinate representation an $n$-dimensional measure polytope in moving coordinate form with side $a$. There are other simpler representations, but this one is needed in the proof of Lemma 2.1.

Lemma 2.2. For any $m, n, r$, and $a$, there is $s$ such that if the $m$-dimensional measure polytopes of side $a$ in $R^{S}$ are $r$-colored, an $n$-dimensional measure polytope of side a in $R^{s}$ exists such that if two of its $m$-dimensional face polytopes are translates, they are the same color.

Proof. Recall Ramsey's theorem states that, for every $n>m$ and every $r$, there is a number $s=\operatorname{Ram}(n, m, r)$ such that if all the $m$-element subsets of an $s$-element set $S$ are colored using $r$ colors, there will be an $n$-element subset of $S$ with all its $m$-element

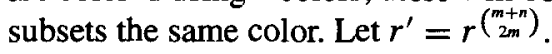


Let $s=\operatorname{Ram}\left(2 n, m+n, r^{\prime}\right)$. Color the $m$-dimensional polytopes of side $a$ of $R^{s}$ with $r$ colors. From this coloring, derive an induced coloring of the $m+n$ sets of a set of $s$ elements with $r^{\prime}$ colors as follows. For every subset $T$ of $m+n$ elements of the $s$-element set $S$, pick the corresponding set $T^{\prime}$ of $m+n$ coordinates of $R^{s}$. Recall that $\left(\begin{array}{c}m+n \\ 2 m\end{array}\right)$ is the number of ways $2 m$ elements can be chosen from an $m+n$ set. Then for each of the $\left(\begin{array}{c}m+n \\ 2 m\end{array}\right)$ sets of $2 m$ elements of $T$, we obtain an $m$-dimensional measure polytope of side $a$ as follows:

First fix a $2 m$-element subset $U$ contained in $T$. Form the set of coordinates, $U^{\prime}$, corresponding to elements in $U$. Then set a coordinate equal to 0 if it is not a member of $T^{\prime}$, and set a coordinate equal to $a / 2^{1 / 2}$ if it is a member of $T^{\prime}$ but not of $U^{\prime}$. From the $2 m$ coordinates of $U^{\prime}$ form $m$ pairs by putting the first two elements in one pair, the third and fourth in another, etc., until we have $m$ pairs. Then let these pairs be the moving pairs. Then, by the previous note, the figure thus obtained is an $m$-dimensional measure polytope in moving coordinate form of side $a, P\left(U^{\prime}\right)$.

Now that we have defined this polytope we return to the problem of the coloring. $P\left(U^{\prime}\right)$ is given one of the $r$ colors by the coloring of $R^{s}$. For each of the $\left(\begin{array}{c}m+n \\ 2 m\end{array}\right)$ choices of $2 m$ elements out of the $m+n$ elements of $T$, we get one of the $r$ colors in this manner. We form the product coloring by assigning to each $(m+n)$-element set the product of each of the colors associated by each choice of $2 m$ elements out of $m+n$ elements. So for each set of $m+n$ elements, we get one of $r^{\prime}$ possible product colorings.

Now that we have defined the coloring we prove the lemma. Because of Ramsey's theorem, we have one $2 n$-element set $V$ with all its $(m+n)$-element subsets monochromatic. Let $V^{\prime}$ be the $2 n$ coordinates corresponding to $V$. Form $n$ pairs from the $2 n$ elements of $V^{\prime}$ as before, pairing the first two elements, then the third and fourth, etc. Denote by $P\left(V^{\prime}\right)$ the $n$-dimensional measure polytope in moving coordinate form of side $a$ whose vertices are formed by allowing each of these $n$ pairs of coordinates of $V^{\prime}$ to be moving pairs, and setting the rest of the coordinates equal to 0 .

Then we claim that if $L_{1}$ and $L_{2}$ are translates of each other and both are $m$-dimensional face polytopes of $P\left(V^{\prime}\right)$, then they are the same color. We note that it can easily be proved that since $L_{1}$ and $L_{2}$ are translates of each other, they have the same $m$ pairs as moving coordinates. The only difference between them is that some of their fixed coordinates have been moved from the first to the second position (or second to first) in these pairs.

We note exchanging the position of any nonzero coordinate within its pair will not affect the relative position of the moving coordinates and the fixed nonzero coordinates because we have chosen the pairs so that none of the $2 n$ coordinates are between any pair's elements. Then, since the relative position of the moving coordinates and the fixed nonzero coordinates are the same for both $L_{1}$ and $L_{2}$, they correspond to the same choice of $2 m$ elements out of $(n+m)$-element sets. Since the product coloring is constant on these $(n+m)$-element sets and since $L_{1}$ and $L_{2}$ correspond to the same choice of $2 m$ elements out of an $(n+m)$-element set, $L_{1}$ and $L_{2}$ will have the same color. Thus the lemma is proved, and we are ready for the proof of the main theorem.

Proof of Theorem 2.1. We apply Lemma 2.2 to get $K$, a $\operatorname{Ram}(n, m, r)$-dimensional measure polytope in moving coordinate form of side $a$ in which the color of an $m$ dimensional face polytope is the same as any polytope which is a translate of it. Then 
the color of an $m$-dimensional face polytope of $K$ depends only on which $m$ of the $\operatorname{Ram}(n, m, r)$ pairs are chosen as moving coordinate pairs.

From this, we get an induced coloring of the $m$ subsets of a $\operatorname{Ram}(n, m, r)$-set. We assign an $m$ subset the color of an $m$-dimensional measure polytope which has $m$ moving coordinate pairs corresponding to the $m$ subset. By Ramsey's theorem, there must be a monochromatic $n$ subset, $S$. Let $S^{\prime}$ be the set of $n$ coordinates corresponding to $S$. Let $P\left(S^{\prime}\right)$ be an $n$-dimensional measure polytope which has $n$ moving coordinate pairs corresponding to the set $S^{\prime}$ and the rest arbitrarily fixed. We show all the $m$-dimensional face polytopes of $P\left(S^{\prime}\right)$ are monochromatic by showing an arbitrary pair of these polytopes are the same color. Let this arbitrary pair be $M_{1}$ and $M_{2} . M_{1}$ and $M_{2}$ have the same color as their corresponding $2 m$-element sets. These $2 m$-element sets are in $S$ since $M_{1}$ and $M_{2}$ are in $P\left(K^{\prime}\right)$. Since these sets are in $S$ they are the same color and hence $M_{1}$ and $M_{2}$ have the same color.

\section{All Cross Polytopes are Edge-Ramsey}

The $n$-dimensional cross polytope is the $n$-dimensional analog of the octahedron. It can be defined as the convex hull of all points in $R^{n}$ with all coordinates equal to 0 except one and this exceptional coordinate is equal to 1 or -1 .

To simplify matters we prove for any $r$ and $m$ the existence of an $n$ such that if $R^{n}$ is $r$-colored, a monochromatic $m$-dimensional cross polytope of side $2^{(r m+2) / 2}$ exists. This allows the use of coordinates 0 and 1 in the proof. For a proof of side $a$ replace 1 with $a / 2^{(r m+2) / 2}$. To allow all coordinates to have coordinates equal to 0 or 1 we assume that all points are embedded in an $n$-dimensional measure polytope in moving coordinate form of side $2^{1 / 2}$. We recall that there is only one such polytope in $2 n$-dimensional space. We assume that all points are vertices of this polytope.

Now we introduce a representation of the $n$-dimensional cross polytope. Since all points are embedded in a $2^{n}$-dimensional measure polytope in moving coordinate form of side $2^{1 / 2}$. We have $2^{n+1}$ coordinates in $2^{n}$ pairs. Then, for each $i=1,2, \ldots, n$, we construct a pair of points as follows. For $1 \leq j \leq 2^{n}$, we let the $j$ th pair of coordinates of the first point of the $i$ th pair of points equal $(1,0)$ if the binary representation of $j-1$ has $i$ th bit (we count bits from the right) 1 , and $(0,1)$ otherwise. The second point is the complement of the first in that it has 1's where the first has 0 's, etc. We refer to this pair of points as the $i$ th pair of points. If a point is a member of the $i$ th pair of points we say it has index $i$. It is easily verified that these $2 n$ points form an $n$-dimensional cross polytope with side $2^{n / 2}$. Moreover, it is easily seen that any $m$ of these pairs form an $m$-dimensional cross polytope. We give an example.

The six vertices of the three-dimensional cross polytope can be represented as:

$$
\begin{aligned}
& (0,1,0,1,0,1,0,1,1,0,1,0,1,0,1,0), \\
& (1,0,1,0,1,0,1,0,0,1,0,1,0,1,0,1) \text {, both of which have index } 3 . \\
& (0,1,0,1,1,0,1,0,0,1,0,1,1,0,1,0), \\
& (1,0,1,0,0,1,0,1,1,0,1,0,0,1,0,1) \text {, both of which have index } 2 . \\
& (0,1,1,0,0,1,1,0,0,1,1,0,0,1,1,0) \text {, } \\
& (1,0,0,1,1,0,0,1,1,0,0,1,1,0,0,1) \text {, both of which have index } 1 .
\end{aligned}
$$

Now we introduce the notion of type. 
We recall we have assumed that all points are vertices of the unique $n$-dimensional measure polytope in moving coordinate form with side $2^{1 / 2}$ in $2 n$-dimensional space. This allows us to use only 1 and 0 as coordinates. The definition of type can be extended to sets of points, all of whose points are either 0 and an arbitrary constant $a$ by substituting $a$ for 1 in the following definition. Edge $b c$ is assigned a type as follows:

We find the first pair where $b$ and $c$ differ. We note which of the points $b$ or $c$ had coordinates of the form 01 in that pair. We call this point, $f$, the first point. We form a string of binary digits as follows: The first bit will always be a 0 . Recall that the first bit is furthest to the right. If (for $i \geq 2$ ) the $i$ th pair where $b$ and $c$ differ has the coordinates 01 at $f$, then the $i$ th bit of the string will be 0 . If it has coordinates 10 at $f$, the $i$ th bit of the string will be 1 . Thus we obtain an injection from any edge to a string of 0 's and 1's.

We see that if $m$ pairs differ, we have $2^{m-1}$ possible types. Moreover, we note that each $m$-dimensional face polytope in moving coordinate representation contains all possible types of edges with $m$ pairs different, one for each of its main diagonals. We also note that each edge with $m$ pairs different is in exactly one $m$-dimensional face polytope.

Lemma 3.1. Suppose $b$ is a member of the $i$ th pair of points, $c$ is a member of the $j$ th pair, and $d$ is a member of the $k$ th pair, with $k>j>i$. Then the edge db and the edge $d c$ will be of the same type. In other words, between points belonging to different pairs, the type is determined solely by the maximum index of the two pairs.

Proof. Assume $d$ starts with $2^{k} 01$ 's followed by the same number of 10 's, etc. Then if $k>i$ and $b$ is a member of the $i$ th pair, the edge $b d$ will consist of (ignoring pairs that are the same in both) $2^{k-1}$ pairs going from 10 to 01 followed by the same number of pairs going from 01 to 10 . This pattern continues in the same manner until all pairs are used, with exactly half the pairs remaining the same in both points.

An example will help make this clear. Assume

$$
d=(0,1,0,1,0,1,0,1,1,0,1,0,1,0,1,0)
$$

which has index 3 . Then if

$$
c=(0,1,0,1,1,0,1,0,0,1,0,1,1,0,1,0)
$$

which has index 2 or if

$$
c=(1,0,0,1,1,0,0,1,1,0,0,1,1,0,0,1)
$$

which has index 1 , the type of $d c$ will be 1100 .

Lemma 3.2. For all $m, k$, and $r$, there is $u=f(k m, r)$ such that if the $u$-dimensional measure polytope $P$ in moving coordinate form of side a as above has its edges of length $d=a(m)^{1 / 2} r$-colored, there is a $k$-dimensional face polytope of side a such that all its diagonals of length d have the property that two edges with the same type have the same color. 
Proof. We give the $m$-dimensional measure polytopes whose vertices are vertices of $P$ the following coloring: We look at its $2^{m-1}$ major diagonals and give each $m$-dimensional polytope the product coloring of its diagonals as follows. Each type which has $m$ moving coordinates corresponds to one of $2^{m-1}$ different binary strings. Treating these strings as numbers we order them. Each $m$-dimensional measure polytope has its main diagonals corresponding to this set of binary numbers. We get the product coloring as follows. We get the first color from the main diagonal corresponding to the lowest binary number, the second color from the diagonal corresponding to the second lowest binary number. We continue in this manner until we have an $r^{2^{m-1}}$-coloring of the $m$-dimensional measure polytopes.

If we make $u$ large enough, there is a $k$-dimensional measure polytope $L$, formed by fixing in some way $u-k$ of the pairs, that has all its $m$-dimensional face polytopes the same color. This follows from Theorem 2.1. Then $L$ satisfies the conditions of the theorem.

Theorem 3.3. All cross polytopes are edge-Ramsey.

Proof. Use Lemma 3.2 (set $a=2^{1 / 2} m=2^{(r n+1)}, d=2^{r n+2) / 2}$ to have all coordinates equal to 1 or 0 ) to get an $m$-dimensional measure polytope, $P$, such that all of its diagonals of length $d$ with $2^{(r n+1)}$ pairs differing have the color determined solely by their type. Then we use the above representation of a cross polytope to get an $(r n+1)$-dimensional cross polytope $K$ whose vertices are a subset of the vertices of $P$ and whose edges are edges diagonals of $P$ of length $d$. Since the edges of $K$ are all diagonals of $P$ with length $d$ their color is solely determined by their type. By Lemma 3.1 we have $r n+1$ pairs of points indexed from 1 to $r n+1$ such that the type and hence the color of any edge between points in a different pair is determined solely by the maximum index of the two pairs.

Then give $i$ the color of any edge between any point in the $i$ th pair and any point in a lower pair, and use the pigeonhole principle to get $n$ indices with the same color. These $n$ pairs will form a cross polytope $L$. Any edge of $L$ will be given the color corresponding to its type. This color will be the same as that assigned to the index corresponding to that type. In our construction we have every index assigned the same color. Hence $L$ will have all its edges monochromatic and we are done. We can substitute an arbitrary constant for one in this construction to get a cross polytope with any side desired.

A configuration $K$ is super-Ramsey if there is a constant $c$ so that if $n>c \log r$ and $R^{n}$ is $r$-colored, then there is a monochromatic set congruent to $K$. Let $K$ be a configuration, and let $p=p(K)$ denote the minimum radius of a sphere containing $K$. The configuration $K$ is hyper-Ramsey if, for every $\varepsilon>0$, a constant $c$ exists such that if $n>c \log r$ and the surface of the $n$-dimensional sphere of radius $p+\varepsilon$ is $r$-colored, then there is a monochromatic copy of $K$. We note that the given representation of a cross polytope, $K$, can be viewed as a subset of a measure polytope, $P$, with the same circumscribed hypersphere of minimum radius. Then from [11] we can see this implies the cross polytope is hyper-Ramsey, and hence super-Ramsey. 


\section{Polygons Which Are Not Edge-Ramsey}

If $P$ is a regular polygon with $n$ sides, $P$ is not edge-Ramsey unless $n=3,4$, or 6 . The proof of this statement requires the following elementary construction. Divide all line segments into classes, with each class consisting of parallel line segments. A coloring of line segments in which parallel line segments always receive the same color is called a slope coloring. There is a natural bijection, $\pi$, between the set of all slope colorings and the set of all colorings of the points of the unit sphere centered at the origin, in which antipodal points get the color of the line segment connecting them. This bijection stems from the obvious fact that each class of parallel line segments uniquely corresponds to an antipodal pair of points.

The remainder of the argument requires the following result of Graham [15].

Theorem 4.1 [15]. Let $K=\left\{x_{1} \cdots x_{t}\right\}$ be a configuration, and let $c_{1} \cdots c_{t}$ be real numbers, no nonempty subset of which sum to zero. Suppose that summation $\sum_{1}^{t} c_{i} x_{i}=0$, and let $p$ be the minimal possible radius of all spheres on whose surface $K$ can be embedded. Then there is a finite number of colors $r$, such that, for any $n$, the surface of the $n$-dimensional sphere of radius $p$ can be r-colored so that it (the surface) does not contain a monochromatic copy of $K$.

For clarity, we emphasize that the integer $n$ in Theorem 4.1 does not depend on $r$. After $r$ is fixed, $n$ can be chosen arbitrarily large, and we can still find a coloring satisfying the theorem. The following elementary result is stated without proof.

Lemma 4.2. If $K$ is a regular polygon with $n$ sides, the line segments connecting adjacent vertices can be translated so they lie on the line segments connecting the origin to the vertices of a regular polygon of $n$ sides.

Remark. We note for odd polygons to get this result we must use translations along the length of the line segment itself to obtain the inversion of the line segment with respect to either of its endpoints to obtain this result. Hence this result would not be true for vectors.

Lemma 4.3. For any $n$ there is an $r$ such that there is a coloring with $r$ colors of the $m$-sphere for any $m$ that does not contain a monochromatic copy of any configuration which, when antipodes are identified, form a regular polygon with $2 n$ sides when $n>3$.

Proof. If a polygon with $2 n$ sides does not have $n$ of its vertices the same color it will not be monochromatic when colors of antipodes are identified. So we see that the all critical configurations involve $n$ points lying on a maximal regular polygon of $2 n$ sides.

Note that Graham's result implies that there is finite number $r$ such that there is a coloring of the $n$-sphere with $r$ colors for any $n$ that does not contain a monochromatic copy of a configuration whose convex hull contains the origin. We can prove this by noting that the convex hull of a set of points is the set of linear combinations of the set of points by positive coefficients which add up to 1 . If the convex hull of a set of points 
does not contain the origin, one of the support lines of the set separates the origin from the set. It follows that the diameter parallel to that support line forms a semicircle in which the set lies and that only one of the $n$ points of the set can lie. For every possible set of $n$ points which lie on the vertices of a $2 n$-gon and contain the origin in its convex hull, we use Graham's theorem to find a coloring that does not contain a monochromatic copy of it. Then we take the product coloring derived from these colorings. This product coloring does not contain a monochromatic copy of any of these configurations.

The one configuration containing $n$ out of $2 n$ points of the regular polygon of $2 n$ sides remaining is $n$ adjacent points of the regular polygon. When $2 n>6$, we have three adjacent points $p, q$, and $r$, satisfying $p+r-c q=0$ with $c>0$ and $c$ not equal to 1 or 2 as long as $2 n$ does not equal 6 . From Graham's theorem an $r$-coloring exists which does not contain a monochromatic copy of this configuration. The product of this coloring with the product coloring produced earlier gives a coloring which does not contain a monochromatic copy of any set of $n$ points which, through identification of antipodes, produces a regular polygon of $2 n$ sides when $2 n>6$.

Theorem 4.4. The pentagon and regular polygons with seven or more sides are not edge-Ramsey.

Proof. Under the identification, $\pi$, described previously, this is equivalent to finding a coloring that does not contain a monochromatic copy of the regular polygon of $n$ sides in the unit sphere, when colors of antipodes are identified. Since a slope coloring is invariant under translation, Lemma 4.2 implies that if we can find a slope coloring without a monochromatic copy of the center of a polygon of $n$ sides connected to its $n$ vertices, we will also have a slope coloring with no monochromatic copies of the edges of the regular polygon of $n$ sides. Lemma 4.3 gives us such a coloring if $n$ is even and greater than six. We note that the line segments connecting the center of a regular polygon of an odd number of sides $2 m+1$ together with the inversion of these segments through the center of a regular polygon of $4 m+2$ sides to its vertices. So any slope coloring which does contain a monochromatic copy of the regular polygon of $4 n+2$ sides will not contain a monochromatic copy of a regular polygon of $2 n+1$ sides.

This observation and the comments made above imply that the only possible polygons which can be edge-Ramsey are those with three, four, or six sides.

We use the machinery of this proof to answer a question in [16]. Is there a finite configuration of points $T$ such that if $R_{t}$ is the minimum radius of a sphere containing $T$ for any $r$, is there an $n$ such that if the sphere of dimension $n$ and radius $R_{t}$ is given any $r$-coloring it will contain a monochromatic copy of $T$ ? We answer in the affirmative that there is such a $T=\left\{(1,0),\left(1 / 2,3^{1 / 2} / 2\right),\left(1 / 2,-3^{1 / 2} / 2\right)\right\} . T$ is isomorphic to three adjacent vertices of the hexagon of radius 1 .

From what has been shown above we know that a slope coloring excluding the regular hexagon would exclude the triangle, but the triangle is edge-Ramsey, so there cannot be such a slope coloring. However, for every set of three vertices, $K$, of the hexagon, which under identification of antipodes forms a hexagon and which contains the origin in its convex hull, there is a number $r$ such that, for all $m$, the unit $m$-sphere can be $r$-colored 
such that there is no monochromatic copy of $K$. We can form a product coloring as we have done above so that there is a finite number $r$ such that, for all $m$, the unit $m$-sphere can be $r$-colored and there is no monochromatic copy of any such configuration. If we could find a coloring that excluded three consecutive vertices of a hexagon, $T$, we could find a slope coloring that excluded the hexagon would not be edge-Ramsey. We have proved above that the triangle is edge-Ramsey.

So $T$ which can be given coordinates $T=\left\{(1,0),\left(1 / 2,3^{1 / 2} / 2\right),\left(1 / 2,-3^{1 / 2} / 2\right)\right\}$ will occur for all possible $r$-colorings of the unit sphere since $T$ is isomorphic to three consecutive vertices of the hexagon. Thus $T$ is the first known configuration having the property that, for any $r$, there is an $n$, such that if the sphere of minimal radius containing $T$ of $n$-dimensions is $r$-colored, it contains a monochromatic copy of $T$.

\section{Miscellaneous Results on Edge-Ramsey and Related Configurations}

In this section we give an example illustrating the techniques and results developed thus far.

Theorem 5.1. The three-point configuration, consisting of two points a and $b$ together with the midpoint $c$ of the line segment $a b$ and the edges ac and $c b$, is not edge-Ramsey, although it is bispherical and its edge graph is a bipartite graph.

Proof. On each line, we color edges of length $|a b| / 2$ such that if two edges are adjacent, they have opposite colors. This prevents any monochromatic copy of the three-point configuration from occurring.

Theorem 5.2. It is possible to color all equilateral traingles in any space with two colors such that no octahedron has all its face triangles the same color.

Proof. We note that the faces of the octahedron can be divided into four pairs such that each pair contains two triangles that lie on parallel planes. These two parallel triangles can be interchanged by a $180^{\circ}$ rotation about the centroid. We also note that, in any $n$-dimensional space, we can two-color triangles such that no triangle is the same color as a triangle which can be made into a translate of it by a $180^{\circ}$ rotation about its centroid.

Theorem 5.3. An edge-configuration $(K, E)$ exists whose endpoints form a nonspherical set which is edge-Ramsey.

Proof. We have already found a triangle (mentioned at the end of the previous section) $T$ so that for any $r$ there is an $n$ such that when the $n$-dimensional sphere at the origin of minimum radius $d$ which contains $T$ is $r$-colored, it contains a monochromatic triangle congruent to $T$.

We simply look at all edges connecting the origin to the $n$-dimensional sphere and give each point of the sphere the color of the edge connecting it to the origin. Then we 
will have a monochromatic triangle and a monochromatic set of edges connecting the origin to the triangle. We now show that the set of points forming the triangle and the origin is not spherical.

The origin is on the same plane as the triangle; otherwise, it would not be the center of the minimum sphere containing the triangle. Let the origin and the points of the triangle lie on some sphere $C$. Then the center of $C$ must be connected to the origin by a line perpendicular to the plane containing the triangle. Let the distance from the center of the sphere $C$ to the origin be $q$. Then the radius of the sphere $C$ must also be $q$. However, the distance from the center of the sphere to a point of the triangle will be $\left(d^{2}+q^{2}\right)^{1 / 2}$, which will be greater than $q$, giving a contradiction.

The following result is trivial and is stated only for emphasis.

Theorem 5.4. If a configuration is edge-Ramsey under congruency transformations, it is edge-Ramsey under similarity transformations.

Finally we use the previous theorems to determine which regular polytopes are edgeRamsey. It was already known that the $n$-dimensional simplices were edge-Ramsey. In the first section we have shown that $n$-dimensional measure polytopes are edge-Ramsey. In the second section we have shown that the $n$-dimensional cross polytopes were edgeRamsey. Finally, the results in the third section prevent all the regular polygons from being edge-Ramsey, except the triangle, the square, and the hexagon. From [4] we recall that the $n$-cell is the regular polytope with $n$ solid faces. Since the pentagon is not edge-Ramsey, the icosahedron, the dodecahedron, the 600-cell, and the 120-cell are not edge-Ramsey, as they all contain pentagons.

There are two cases left: the hexagon and the 24-cell. It remains unknown whether these two configurations are edge-Ramsey.

\section{References}

1. T. F. Banchoff, Beyond the Third Dimension, Scientific American Library, New York (1990).

2. G. J. Butler, Simultaneous packing and covering in Euclidean space, Proc. London Math. Soc. 3 (1972), 721-735.

3. K. Cantwell, Euclidean Ramsey Theory, Ph.D. Thesis, Arizona State University (1992).

4. H. S. M. Coxeter, Regular Polytopes, Macmillan, New York (1963).

5. P. Erdös, R. L. Graham, P. Montgomery, B. L. Rothschild, J. Spencer, and E. G. Straus, Euclidean Ramsey theorems, 1, J. Combin. Theory 14 (1973), 341-363.

6. P. Erdös, R. L. Graham, P. Montgomery, B. L. Rothschild, J. Spencer, and E. G. Straus, Euclidean Ramsey theorems, II, in Finite and Infinite Sets, Colloq. Math. Soc. János Bolyai, North-Holland, Amsterdam (1973), pp. 529-558.

7. P. Erdös, R. L. Graham, P. Montgomery, B. L. Rothschild, J. Spencer, and E. G. Straus, Euclidean Ramsey theorems, III, in Finite and Infinite Sets, Colloq. Math. Soc. János Bolyai, North-Holland, Amsterdam (1973), pp. 559-583.

8. P. Erdös and C. A. Rogers, Covering space with convex bodjes, Acta Arith. 7 (1962), 281-285.

9. P. Frankl and V. Rödl, All triangles are Ramsey, Trans. Amer. Math. Soc. 29 (1980), 777-779.

10. P. Frankl and V. Rödl, Forbidden intersections, Trans. Amer. Math. Soc. 30 (1987), 249-255.

11. P. Frankl and V. Rödl, A partition property of simplices in Euclidean space, J. Amer. Math. Soc. 3 (1990), 1-7. 
12. P. Frankl and I. G. Rosenberg, A finite set intersection theorem, European J. Combin. 2 (1981), 127-129.

13. P. Frankel and R. M. Wilson, Intersection theorems with geometric consequences, Combinatorica 4 (1981), 357-368.

14. R. L. Graham, Rudiments of Ramsey Theory, American Mathematical Society, Providence, RI (1980).

15. R. L. Graham, Euclidean Ramsey theorems in the $n$-sphere, J. Graph Theory 7 (1983), 105-114.

16. R. L. Graham, Old and new Euclidean Ramsey theorems, in Discrete Geometry and Convexity, Ann. New York Acad. Sci., vol. 440 (1985), pp. 20-30.

17. R. L. Graham, Topics in Euclidean Ramsey theory, in Mathematics of Ramsey Theory, Springer-Verlag, New York (1990), pp. 200-213.

18. R. L. Graham, B. L. Rothschild, and J. Spencer, Ramsey theory, 2nd edn., Wiley, New York (1990).

19. B. Grünbaum, Convex Polytopes, Interscience, London, (1967).

20. R. Juhász, Ramsey type theorems in the plane, J. Combin. Theory Ser. A 27 (1979), 132-160.

21. I. Kř́ž , Permutation groups in Ramsey theory, Preprint.

22. D. G. Larman and C. A. Rogers, The realization of distances within sets in Euclidean space, Mathematika 19 (1972), 1-24.

23. P. Pearce and S. Pearce, Polyhedra Primer, Van Nostrand Reinhold, New York (1978).

24. H. S. Promel and Bernd Voigt, Aspects of Ramsey Theory I. Sets II. Arithmetic Progressions III. Parameter Sets, Fasind Institute for Diskrete Mathematics, Bonn (1989).

25. V. Rödl, On a problem in combinatorial geometry, Discrete Math. 45 (1983), 129-131.

26. L. E. Shader, All right triangles are Ramsey in $E^{2}, J$. Combin. Theory Ser. A 20 (1976), 385-389.

27. L. A. Székely and W. C. Wormald, Bounds on the measurable chromatic number of $R^{n}$, Discrete Math. 75 (1989), 343-372.

28. D. R. Woodall, Distance realized by sets covering the plane, J. Combin. Theory Ser. A 14 (1973), 187-200.

Received September 5, 1994, and in revised form February 21, 1995, March 16, 1995, and April 17, 1995. 